\title{
Longitudinal Beam-Shaping Simulation for Enhanced Transformer Ratio in Beam-Driven Accelerators
}

\author{
Wei Hou TAN \\ Northern Illinois University \\ DeKalb, Illinois, U.S.A. \\ z1829753@ students.niu.edu
}

\author{
Philippe PIOT \\ Fermi National Accelerator Laboratory \& \\ Northern Illinois University \\ DeKalb, Illinois, U.S.A.
}

\author{
Alexander ZHOLENTS \\ Advanced Photon Source \\ Argonne National Laboratory \\ Lemont, Illinois, U.S.A.
}

\begin{abstract}
A beam-driven structure wakefield accelerator (SWFA) is currently under consideration for future multi-user $\mathrm{X}$ ray free-electron-laser (FEL) light sources. One of the challenges associated with SWFA is the efficient energy transfer from the drive to the main bunch that can be enhanced via a precise control over the drive-bunch current profile. This paper discusses the development and initial beam-dynamics simulation of the drivebunch longitudinal dynamics using the fast-tracking program TWICE. This code, written in PYTHON, allows for rapid prototyping of the beam shaping process while taking into account the collective effects using simplified models. This paper describes the algorithm implementation and its capabilities of including collective effects into both forward and backward tracking. Initial results of optimizing beam shape with a conventional linac to produce a drive beam with a shape optimized for SFWA are presented.
\end{abstract}

Index Terms-beam-driven wakefield accelerators, longitudinal beam dynamics, collective effects

\section{INTRODUCTION}

A critical figure of merit associated with Structure-based Beam-driven Wakefield Acceleration (SWFA) is the transformer ratio $\mathcal{R}$, defined as the ratio of the maximum of the accelerating field behind the bunch $E_{+}$, to the maximum of the decelerating field within the bunch $E_{-}$,

$$
\mathcal{R}=\frac{\max \left|E_{+}\right|}{\max \left|E_{-}\right|},
$$

as shown in Fig. 1. For a symmetric current distribution, $\mathcal{R} \leq$ 2 according to the beam loading theorem. However, it was recognized that tailoring the current distribution could enhance the transformer ratio to arbitrarily large values [1]. Although such an enhancement, for a given bunch charge, come at the expense of a reduced peak accelerating electric field [2]. Over the years, several beam shaping techniques have been proposed and investigated including techniques based on shaping photocathode lasers ( [3], [4], [5]), transverse-tolongitudinal phase-space emittance exchange [6], and multifrequency linacs [7]. Most of the shaping schemes (except for the phase-space emittance exchange) explore only the longitudinal degree of freedom and can be assisted by collective effects acting in the longitudinal phase space. Therefore, the transverse beam dynamics can be ignored at the early stage of the bunch-shaping optimization process. Consequently, we have written a simple simulation package, Tracking With longItudinal CoordinatE (TWICE), to track the evolution of macroparticles in the longitudinal phase space (LPS). It should be noted that several programs have been developed over the years [8] based on MATLAB. In contrast, TWICE was written using the open-source PYTHON language to leverage the large number of tools available (e.g., optimization and machinelearning algorithms) and to be deployable on large computer clusters for rapid throughput simulations (e.g., as needed for sensitivity studies or optimizations). It should be stressed that TWICE is not meant to be a full-fledged tracking code but is developed to allow rapid prototyping and complement other beam simulation tools. In this paper, we describe TWICE's capabilities and benchmark results against other codes. We also demonstrate the backward-tracking capabilities taking the example of a final asymmetric-doorstep distribution [1].

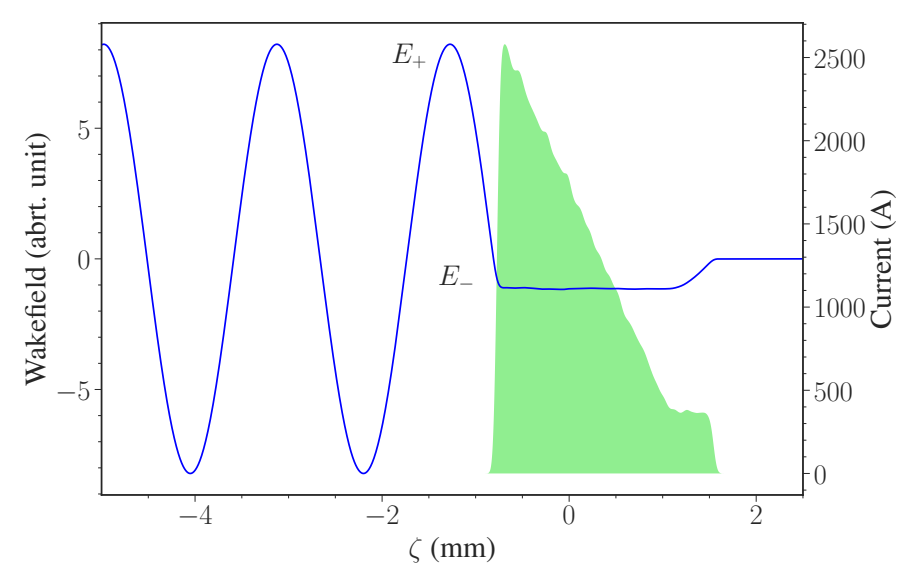

Fig. 1. The longitudinal wakefield (blue trace) generated by a doorstep bunch distribution (green-shaded area) as it passes through a dielectric waveguide. The achieved transformer ratio is $\mathcal{R} \approx 6$.

\section{LONGitudinal Tracking Code, TWICE}

The design and implementation of TWICE can be categorized into two parts, beam distribution and beamline

This manuscript has been authored by Fermi Research Alliance, LLC under Contract No. DE-AC02-07CH11359 with the U.S. Department of Energy, Office of Science, Office of High Energy Physics. 
elements. The beam is represented by a collection of macroparticles with user-defined total charge and LPS coordinate $(\zeta, E)$. We take $\zeta$ to be the longitudinal position with respect to a reference particle (defined by the user or assigned to be the bunch barycenter) and $E$ to be the total energy. Our sign convention is such that $\zeta>0$ corresponds to the bunch's head.

The initial bunch distribution can be generated internally using the standard PYTHON random generator library, a Monte-Carlo generator, or loaded from an external file, e.g., from the ASTRA or IMPACT programs. Likewise, we consider implementing an alternative particle generator algorithm that is free from numerical noise, similar to [9], [10].

\section{A. Beamline elements}

The longitudinal dynamics of the beam is modeled by a simple LPS coordinate transformation $\left(\zeta_{i}, E_{i}\right) \rightarrow\left(\zeta_{f}, E_{f}\right)$. The acceleration through a radiofrequency $(\mathrm{RF})$ linac is described by the transformation:

$$
\begin{aligned}
\zeta_{f} & =\zeta_{i}, \\
E_{f} & =E_{i}+e V_{\mathrm{RF}} \cos \left(\phi-\frac{2 \pi f}{c} \zeta_{i}\right),
\end{aligned}
$$

where $V_{\mathrm{RF}}$ is the accelerator voltage, $\phi$ is the off-crest acceleration phase, and $f$ is the rf linac frequency. Likewise, an arbitrary longitudinal transformation in a dispersive section is described via

$$
\begin{aligned}
\zeta_{f} & =\zeta_{i}+R_{56} \delta_{i}+T_{566} \delta_{i}^{2}, \\
E_{f} & =E_{i},
\end{aligned}
$$

where $\delta_{i} \equiv \frac{E_{i}-E_{\text {ref }}}{E_{\text {ref }}}$ is the relative momentum offset [here $E_{\text {ref }}$ is a reference energy which can be the energy of the reference particle or an arbitrary energy (e.g., to model off-reference energy dynamics)], and $R_{56} \equiv \frac{\partial \delta}{\partial \zeta}$ and $T_{566} \equiv \frac{\partial^{2} \delta}{\partial \zeta^{2}}$ are the linear and nonlinear longitudinal dispersions, respectively.

In addition to these simple models, a transformation describing a four bend-chicane is also introduced.

\section{B. Collective effects}

Collective effects arising from the interaction of electrons within the beam or from the interaction with external structures can significantly affect the beam dynamics. The collective effects implemented in TWICE include wakefield, coherent synchrotron radiation (CSR), and longitudinal space charge (LSC). In particular, the numerical calculation of these collective effects involves estimating the charge density of the beam, calculating the convolution integral of Green's function with the charge density. In TWICE, longitudinal treatment of collective effects is implemented as a beamline element's operation, specifically energy kick on beam $\Delta E\left(\zeta_{i}\right)$ with $E_{i}\left(\zeta_{i}\right) \rightarrow E_{f}\left(\zeta_{i}\right)=E_{i}\left(\zeta_{i}\right)+\Delta E\left(\zeta_{i}\right)$ and $\zeta_{i} \rightarrow \zeta_{f}=\zeta_{i}$.
1) Charge Density Estimation: One of the challenges in computing collective effects regards the calculation of the current distribution associated with an ensemble of macroparticles (rather than electrons). The macroparticle representation yields numerical noise that has to be suppressed. In TWICE the current distribution can be computed using a standard histogram-binning method combined with a filtering algorithm to remove high-frequency noise, or using the kernel density estimation (KDE) technique [11].

2) Wakefield: The longitudinal wake potential is calculated via a convolution integral of Green's function $w_{z}$ and longitudinal charge density $\Lambda(\zeta)$. The wake function can be obtained from a user-defined function or loaded from an external data file. The integral is shown below:

$$
\begin{gathered}
W(\zeta)=\int_{-\infty}^{\zeta} \Lambda\left(\zeta^{\prime}\right) w_{z}\left(\zeta-\zeta^{\prime}\right) \mathrm{d} \zeta^{\prime} \\
\text { where } \int_{-\infty}^{\infty} \Lambda\left(\zeta^{\prime}\right) \mathrm{d} \zeta^{\prime}=\text { total charge of a bunch, }
\end{gathered}
$$

and the rate of energy change is given by $\frac{d E}{d c t}=W(\zeta)$, where ct represents the active length over which the wakefield affects the beam. In the ultra-relativistic regime, the particle longitudinal dynamics is frozen (so that the relative longitudinal position $\zeta$ does not change in non-dispersive sections). Consequently, an impulse approximation can be used where the effect of the wakefield is locally applied as an energy kick of the form $\Delta E(\zeta)=\int_{c t_{0}}^{c t_{1}} c W(\zeta) d t \simeq L W(\zeta)$, where $L \equiv c\left(t_{1}-t_{0}\right)$ represents the effective length over which the beam is subjected to the wakefield. Several built-in Green's functions $w_{z}(\zeta)$ are available in TWICE [e.g., empirically-parameterized geometric wakefield in TESLA superconducting cavities, simple harmonic wakefield of the form $\left.w_{z}(\zeta)=w_{0} \cos (\zeta)\right]$. Likewise, Green's functions associated with more complicated structures can be directly imported from an external file.

3) Coherent Synchrotron Radiation (CSR): CSR in dipole magnets is calculated using a one-dimension model [12] commonly used in other beam-dynamics codes, such as IMPACT and ELEGANT. To simplify the calculation and speed up the simulation, only steady-state CSR is currently implemented in TWICE. Given a bunch with longitudinal charge density $\Lambda(\zeta)$, the steady-state integral of CSR is given by [13],

$$
\begin{aligned}
& I_{c s r}\left(\zeta, \zeta^{\prime}\right)=-\gamma \frac{e}{4 \pi \varepsilon_{0} R} \frac{4 u\left(u^{2}+8\right)}{\left(u^{2}+4\right)\left(u^{2}+12\right)}, \\
& \frac{\gamma^{3}\left(\zeta-\zeta^{\prime}\right)}{R}=\frac{u^{3}}{24}+\frac{u}{2}, \\
& \frac{d E}{d c t}=\int_{-\infty}^{\zeta} \frac{\partial \Lambda\left(\zeta^{\prime}\right)}{\partial \zeta^{\prime}} I_{c s r}\left(\zeta, \zeta^{\prime}\right) \mathrm{d} \zeta^{\prime},
\end{aligned}
$$

where $\gamma, e, \varepsilon_{0}, R$, and $\theta$ are Lorentz factor, electron charge, permittivity of free space, bending radius, and angle, respectively. CSR is treated as an energy kick localized downstream of the bending element (in a similar fashion to 
that described for the wakefield calculation). For example, a CSR kick with user-defined radius and angle can be included directly downstream of a dipole magnet described by its $R_{56}$.

4) Longitudinal Space Charge (LSC): LSC is also implemented using a one-dimensional (1D) model described by the longitudinal impedance [14]

$$
Z(k)=i \frac{Z_{0}}{\pi \gamma r_{b}} \frac{1-2 I_{1}\left(\xi_{b}\right) K_{1}\left(\xi_{b}\right)}{\xi_{b}},
$$

where $\xi_{b} \equiv k r_{b} / \gamma ; I_{1}$ and $K_{1}$ are modified Bessel functions of the first and second kind, respectively; and $k$ and $r_{b}$ are, respectively, the wavevector amplitude and a user-defined transverse radius. The energy kick is therefore computed in the frequency domain as $\Delta \tilde{E}(k)=Z(k) \tilde{I}(k)$, where $\tilde{I}(k) \equiv \mathcal{F}[I(\zeta)]$ is obtained from a fast-Fourier-transform (FFT) algorithm from the computed current distribution. The final energy kick $\Delta E(\zeta)=\mathcal{F}^{-1}[\Delta \tilde{E}(k)]$. In its current implementation, LSC can be included as a standalone LSC kick in drift spaces and linacs. The LSC calculation in a linac for one linac section is lumped into one step and uses an effective Lorentz factor defined as the geometric average $\gamma_{\text {eff }}=\sqrt{\gamma_{\mathrm{i}} \gamma_{\mathrm{f}}}$, where $\gamma_{\mathrm{i}, \mathrm{f}}$ are the Lorentz factors at the entrance and exit of the linac section, respectively.

\section{Backward tracking}

In TWICE, once a beamline is specified, a final distribution can be tracked in the backward direction. Such a time-reversal feature is convenient to search for an optical accelerator configuration capable of providing a given beam-current distribution [3]. In the ultra-relativistic regime the reverse tracking can be implemented via inverted transformation for a linac

$$
\begin{aligned}
\zeta_{i} & =\zeta_{f}, \\
E_{i} & =E_{f}-e V_{\mathrm{RF}} \cos \left(\phi-\frac{2 \pi f}{c} \zeta_{f}\right) .
\end{aligned}
$$

Similarly, in a longitudinal map, $\delta_{f}=\delta_{i}$ implies

$$
\begin{aligned}
\zeta_{i} & =\zeta_{f}-R_{56} \delta_{f}-T_{566} \delta_{f}^{2}, \\
E_{i} & =E_{f} .
\end{aligned}
$$

Since all collective effects in TWICE are treated as energy kicks, one can flip the sign of such energy kicks to include them into backward tracking $E_{i}\left(\zeta_{i}\right)=E_{f}\left(\zeta_{f}\right)-\Delta E\left(\zeta_{i}\right)$ (again since $\zeta_{f}=\zeta_{i}$ ). Such an approximation is not strictly exact, but it provides a good starting point to estimate the desired initial distribution, which can then be included into more sophisticated tracking codes to optimize the accelerator design.

\section{BENCHMARK RESULTS}

This section provides examples of validations of our algorithms against ELEGANT and published data.

\section{A. LSC from a density modulated bunch}

We consider an example from section II B of J. Qiang et al. [14], where a uniform beam distribution with current 120 A, energy $120 \mathrm{MeV}$, and transverse radius $r_{b}=200 \mu \mathrm{m}$, is perturbed by a $5 \%$ modulation. Its current profile is given by

$$
I_{\text {modulated }}=I_{0}\left(1+0.05 \cos \frac{2 \pi \zeta}{\lambda}\right),
$$

where $I_{0}=120 A$ and $\lambda=15 \mu \mathrm{m}, 30 \mu \mathrm{m}, 50 \mu \mathrm{m}$. We generate 100,000 macroparticles with total bunch length $=10 \lambda$ from Eq. (16) and propagate through a 3-meter drift space. Results shown here in Fig. 2 below agree with Fig. 2 of [14].

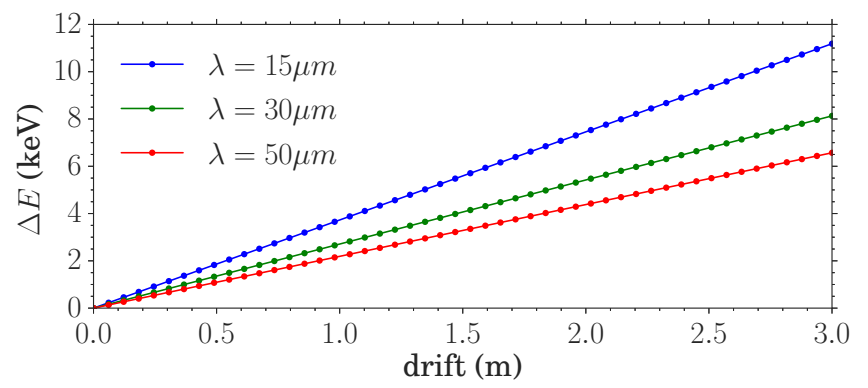

Fig. 2. Amplitude of energy modulation as a function of drift space distance for a bunch described by Eq. (16).

\section{B. Acceleration with geometric wakefield}

We now consider the case of a $10-\mathrm{nC}, 30-\mathrm{MeV}$ bunch injected in a 8.29-m TESLA accelerating cryomodule (consisting of eight TESLA cavities) providing a total accelerating voltage $V_{\mathrm{RF}}=100 \mathrm{MV}$ with phase $\phi=10^{\circ}$. The bunch current follows a Gaussian distribution $\sigma_{z}=0.53 \mathrm{~mm}$. In TWICE the bunch is represented with 100,000 macroparticles. The geometric wakefield associated with a TESLA cryomodule [15] is included in our simulations. Figure 3 confirms that the output LPS computed from TWICE is in agreement with the one obtained from ELEGANT.
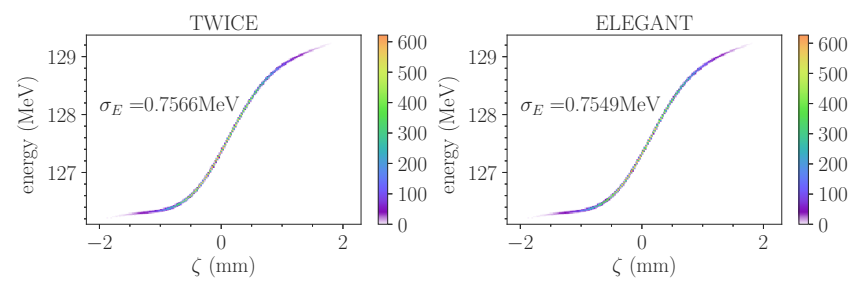

Fig. 3. Comparison of final LPS generated via TWICE (left) and ELEGANT (right) for a $30-\mathrm{MeV}, 10-\mathrm{nC}$ bunch after $10^{\circ}$ off-crest acceleration at $100 \mathrm{MV}$ in a TESLA cryomodule taking into account the geometric wakefield.

\section{Bunch Shaping With Conventional Linac to ENHANCE TRANSFORMER RATIO}

Beam-driven SWFA is a potential candidate for future light sources because of its high gradient acceleration with small physical footprint. Recent studies show that there is a tradeoff between the accelerating gradient and the transformer 
ratio [2]. In addition, the drive bunch is subject to beam-breakup (BBU) instability. The BBU instability can be circumvented by imparting a correlated energy spread on the bunch to provide Balakin-Novokhatsky-Smirnov (BNS) damping [16]. In the context of SWFA a modified criterion is discussed in Ref. [17] and typically requires a substantial $(\sim 10 \%)$ correlated fractional energy spread be maintained through the entire deceleration of the drive bunch.

Producing such a drive bunch with the required beam current profile and LPS correlations involves fine tuning of the electron source and beam shaping linac parameters. While optimization algorithms such as multi-objective genetic algorithms (MOGA) are prevalent, it can be time-consuming without a prior knowledge of a good starting point for parameters. Instead of optimizing the whole beamline starting from the electron gun throughout the linac, we split the effort into two parts, bunch shaping in an electron gun and in a conventional linac. Beam simulation in an electron gun requires a fullfledged simulation code since it is in the low energy regime, where space-charge effects in a 10 -nC bunch play a significant role. In contrast, beam shaping in a conventional linac can be initially performed with the simplified longitudinal-tracking methods implemented in TWICE.

We now consider a specific example where a conventional linac is used to tailor the bunch distribution. A desirable current profile for use in SWFA, assuming a single-mode wakefield excitation there, is the doorstep distribution (see Fig. 1) given by

$$
I(\zeta)= \begin{cases}I_{0}\left(1+\frac{2 \pi}{\lambda}\left(\zeta_{\text {door }}-\zeta\right)\right) & \text { if } \zeta_{\text {min }} \leq \zeta<\zeta_{\text {door }}, \\ I_{0} & \text { if } \zeta_{\text {door }} \leq \zeta \leq \zeta_{\max }, \\ 0 & \text { elsewhere }\end{cases}
$$

where $\lambda$ is the wavelength of the excited mode in the considered SWFA, and $I_{0}$ is the current at the bunch head.

Specifically, we focus on achieving a final distribution with peak current $I_{\text {peak }} \approx 3.5 \mathrm{kA}$ to excite wakefields in a corrugated waveguide with the fundamental mode centered at $220 \mathrm{GHz}$ $(\lambda=1.335 \mathrm{~mm})$. We further assume the injection energy in the SWFA is $400 \mathrm{MeV}$. Additionally, the stability condition discussed in Refs. [17], [18] imposes a total correlated fraction energy spread of $4 \sigma_{\delta}=0.1$. The fluctuations observed in Fig. 1 are due to a numerical noise that we consider reducing by employing a particle generator free from numerical noise. It will allow us to reduce the number of macroparticles and hence speed up the simulations.

We consider a 10-nC final distribution with profile described by Eq. (17) (with $\lambda=1.335 \mathrm{~mm}$ ) at $400 \mathrm{MeV}$. The distribution is represented by 100,000 macroparticles, and the associated LPS appears in Fig. 4. We perform a backward-tracking simulation in the beamline, shown in Fig. 5, with parameters gathered in Tab. I. The example beamline follows a widely adopted linac architecture used in XFELs. The accelerator combines two TESLA-type superconducting accelerating cryomodules (shown as L1 and L2) with a third-harmonic linac (L39). The bunch compression is accomplished in two stages at $102 \mathrm{MeV}$ (BC1) and $400 \mathrm{MeV}$ (BC2). A gun, possibly combined with a short linac, would precede L1.
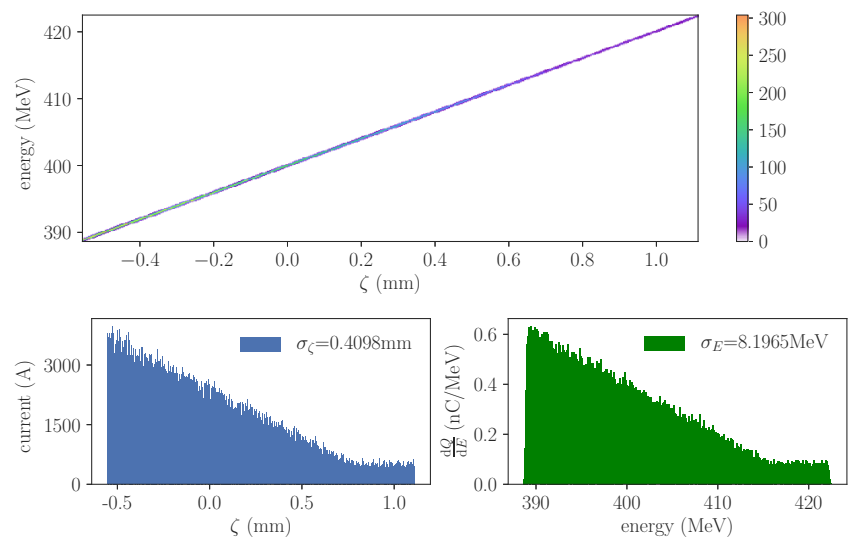

Fig. 4. Targeted final LPS distribution (top) with corresponding longitudinal (bottom left) and energy (bottom right) distributions. The bunch charge is $10 \mathrm{nC}$, and $\zeta>0$ is associated with the bunch head.

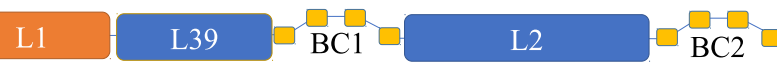

Fig. 5. Diagram of the linac used in the simulations to produce the tailored bunch. L1 and L2 are 1.3-GHz linacs, L39 is a $3.9-\mathrm{GHz}$ linac, and $\mathrm{BC} 1$ and $\mathrm{BC} 2$ are magnetic bunch compressors.

TABLE I

ACCELERATOR SETTINGS USED FOR BACKWARD-TRACKING SIMULATIONS.

\begin{tabular}{|l|c|c|}
\hline Parameter & Value & Unit \\
\hline Accelerating voltage L1 & 100 & $\mathrm{MV}$ \\
\hline Phase L1 & 42 & $\mathrm{deg}$ \\
\hline Frequency L1 & 1.3 & $\mathrm{GHz}$ \\
\hline Accelerating voltage L39 & 30 & $\mathrm{MV}$ \\
\hline Phase L39 & -195 & $\mathrm{deg}$ \\
\hline Frequency L39 & 3.9 & $\mathrm{GHz}$ \\
\hline$R_{56}$ BC1 & -0.05 & $\mathrm{~m}$ \\
\hline$T_{566}$ BC1 & 0.1 & $\mathrm{~m}$ \\
\hline Accelerating voltage L2 & 300 & $\mathrm{MV}$ \\
\hline Phase L2 & -5 & $\mathrm{deg}$ \\
\hline Frequency L2 & 1.3 & $\mathrm{GHz}$ \\
\hline$R_{56}$ BC2 & -0.03 & $\mathrm{~m}$ \\
\hline$T_{566}$ BC2 & -0.1 & $\mathrm{~m}$ \\
\hline
\end{tabular}

A conventional four-bend chicane compressor has $R_{56}>0$ and requires a negative chirp bunch (the bunch head has lower energy) for compression. However, the stability constraint requires a positive chirp bunch (the bunch head has higher energy). Hence, our model implements negative values of $R_{56}$ to compress the bunch. At the present stage, we are also studying chicane designs that can achieve our purpose, such as discussed in Ref. [19]. Also, note that obtaining the chosen $T_{566}$ would require a precise design of the $\mathrm{BCs}$, most likely including sextupole magnets.

Figure 6 presents the initial LPS obtained from backtracking simulations including LCS, wakefield in all the linacs, and 
CSR effect in the magnetic chicanes. The CSR effect was modeled assuming, for simplicity, that all dipole magnets were $50-\mathrm{cm}$ long with a $2^{\circ}$ bending angle.
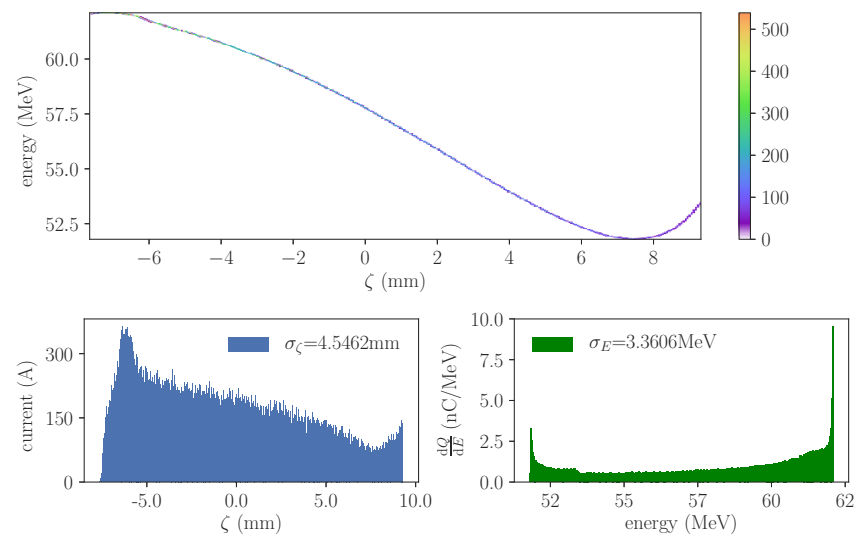

Fig. 6. Resulting initial LPS distribution (top) with associated projections (bottom plots) obtained by the backward-tracking simulation.

\section{SUMMARY}

We have developed the simulation package TWICE and benchmarked it with the known results. We applied it for backward tracking in the linac using the doorstep initial distribution. This package will be further refined and used for defining initial parameters for the accelerator delivering the drive bunches for structure-based wakefield accelerators.

\section{ACKNOWLEDGEMENTS}

This work is supported by the laboratory-directed R\&D program at ANL via the U.S. Department of Energy, Office of Science, under contract No. DE-AC02-06CH11357 and by the U.S. DOE award No. DE-SC0018656 at NIU.

\section{REFERENCES}

[1] K. L. Bane, P. Chen, and P. B. Wilson, "On collinear wake field acceleration," Proceedings of the 1985 Particle Accelerator Conference (PAC1985): Accelerator Engineering and Technology Vancouver, BC May 13-16, 1985, vol. 32, pp. 3524-3526, 1985.

[2] S. S. Baturin and A. Zholents, "Upper limit for the accelerating gradient in the collinear wakefield accelerator as a function of the transformer ratio," Phys. Rev. Accel. Beams, vol. 20, no. 6, p. 061302, Jun. 2017. [Online]. Available: https://link.aps.org/doi/10.1103/PhysRevAccelBeams.20.061302

[3] M. Cornacchia, S. Di Mitri, G. Penco, and A. A. Zholents, "Formation of electron bunches for harmonic cascade x-ray free electron lasers," Phys. Rev. ST Accel. Beams, vol. 9, no. 12, p. 120701, Dec. 2006. [Online]. Available: https://link.aps.org/doi/10.1103/PhysRevSTAB.9.120701

[4] G. Penco, M. Danailov, A. Demidovich, E. Allaria, G. De Ninno, S. Di Mitri, W. M. Fawley, E. Ferrari, L. Giannessi, and M. Trov, "Experimental Demonstration of Electron Longitudinal-Phase-Space Linearization by Shaping the Photoinjector Laser Pulse," Phys. Rev. Lett., vol. 112, no. 4, p. 044801, Jan. 2014. [Online]. Available: https://link.aps.org/doi/10.1103/PhysRevLett.112.044801

[5] F. Lemery and P. Piot, "Tailored electron bunches with smooth current profiles for enhanced transformer ratios in beam-driven acceleration," Phys. Rev. ST Accel. Beams, vol. 18, no. 8, p. 081301, Aug. 2015. [Online]. Available: https://link.aps.org/doi/10.1103/PhysRevSTAB.18.081301
[6] B. Jiang, C. Jing, P. Schoessow, J. Power, and W. Gai, "Formation of a novel shaped bunch to enhance transformer ratio in collinear wakefield accelerators," Phys. Rev. ST Accel. Beams, vol. 15, no. 1, p. 011301, Jan. 2012. [Online]. Available: https://link.aps.org/doi/10.1103/PhysRevSTAB.15.011301

[7] P. Piot, C. Behrens, C. Gerth, M. Dohlus, F. Lemery, D. Mihalcea, P. Stoltz, and M. Vogt, "Generation and Characterization of Electron Bunches with Ramped Current Profiles in a DualFrequency Superconducting Linear Accelerator," Phys. Rev. Lett., vol. 108, no. 3, p. 034801, Jan. 2012. [Online]. Available: https://link.aps.org/doi/10.1103/PhysRevLett.108.034801

[8] K. L. F. Bane and P. Emma, "Litrack: A Fast Longitudinal Phase Space Tracking Code with Graphical User Interface," in Proceedings of the 2005 Particle Accelerator Conference, May 2005, pp. 4266-4268.

[9] B. W. J. McNeil, M. W. Poole, and G. R. M. Robb, "Unified model of electron beam shot noise and coherent spontaneous emission in the helical wiggler free electron laser," Phys. Rev. ST Accel. Beams, vol. 6, no. 7, p. 070701, Jul. 2003. [Online]. Available: https://link.aps.org/doi/10.1103/PhysRevSTAB.6.070701

[10] W. M. Fawley, "Algorithm for loading shot noise microbunching in multidimensional, free-electron laser simulation codes," Phys. Rev. ST Accel. Beams, vol. 5, no. 7, p. 070701, Jul. 2002. [Online]. Available: https://link.aps.org/doi/10.1103/PhysRevSTAB.5.070701

[11] T. A. Mohayai, P. Snopok, D. Neuffer, and C. Rogers, "Novel Application of Density Estimation Techniques in Muon Ionization Cooling Experiment," in Proceedings, Meeting of the APS Division of Particles and Fields (DPF 2017): Fermilab, Batavia, Illinois, USA, July 31 - August 4, 2017, 2017. [Online]. Available: http://lss.fnal.gov/archive/2017/conf/fermilab-conf-17-476-apc.pdf

[12] E. L. Saldin, E. A. Schneidmiller, and M. V. Yurkov, "On the coherent radiation of an electron bunch moving in an arc of a circle," Nuclear Instruments and Methods in Physics Research Section A: Accelerators, Spectrometers, Detectors and Associated Equipment, vol. 398, no. 2, pp. 373 - 394, 1997. [Online]. Available: http://www.sciencedirect.com/science/article/pii/S016890029700822X

[13] C. E. Mitchell, J. Qiang, and R. D. Ryne, "A fast method for computing 1-D wakefields due to coherent synchrotron radiation," Nuclear Instruments and Methods in Physics Research Section A: Accelerators, Spectrometers, Detectors and Associated Equipment, vol. 715, pp. 119 - 125, 2013. [Online]. Available: http://www.sciencedirect.com/science/article/pii/S0168900213002878

[14] J. Qiang, R. D. Ryne, M. Venturini, A. A. Zholents, and I. V. Pogorelov, "High resolution simulation of beam dynamics in electron linacs for x-ray free electron lasers," Phys. Rev. ST Accel. Beams, vol. 12 , no. 10 , p. 100702 , Oct. 2009. [Online]. Available: https://link.aps.org/doi/10.1103/PhysRevSTAB.12.100702

[15] T. Weiland and I. Zagorodnov, "The short-range transverse wake function for TESLA accelerating structure," 2003.

[16] V. E. Balakin, A. V. Novokhatsky, and V. P. Smirnov, "VLEPP: TRANSVERSE BEAM DYNAMICS," Proceedings, 12th International Conference on High-Energy Accelerators, HEACC 1983: Fermilab, Batavia, August 11-16, 1983, vol. C830811, pp. 119-120, 1983.

[17] S. S. Baturin and A. Zholents, "Stability condition for the drive bunch in a collinear wakefield accelerator," Phys. Rev. Accel. Beams, vol. 21, no. 3, p. 031301, Mar. 2018. [Online]. Available: https://link.aps.org/doi/10.1103/PhysRevAccelBeams.21.031301

[18] A. Zholents, W. Gai, S. Doran, R. Lindberg, J. G. Power, N. Strelnikov, Y. Sun, E. Trakhtenberg, I. Vasserman, C. Jing, A. Kanareykin, Y. Li, Q. Gao, D. Y. Shchegolkov, and E. I. Simakov, "A preliminary design of the collinear dielectric wakefield accelerator," Nuclear Instruments and Methods in Physics Research Section A: Accelerators, Spectrometers, Detectors and Associated Equipment, vol. 829, pp. 190 - 193, 2016. [Online]. Available: http://www.sciencedirect.com/science/article/pii/S0168900216001479

[19] A. He, F. Willeke, L. H. Yu, L. Yang, T. Shaftan, G. Wang, Y. Li, Y. Hidaka, and J. Qiang, "Design of low energy bunch compressors with space charge effects," Phys. Rev. ST Accel. Beams, vol. 18, no. 1, p. 014201, Jan. 2015. [Online]. Available: https://link.aps.org/doi/10.1103/PhysRevSTAB.18.014201 